\title{
Síntese e caracterização do material hidrotalcita-hidroxiapatita dopado com nanotubos de carbono e sua aplicação na catálise da reação de transesterificação
}

\section{(Synthesis and characterization of hydrotalcite-hydroxyapatite material doped with carbon nanotubes and its application in catalysis of transesterification reaction)}

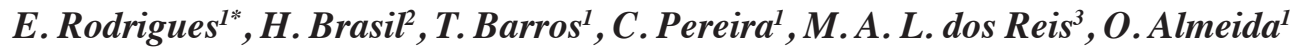 \\ ${ }^{1}$ Universidade Federal do Pará, Faculdade de Química, 66075-110, Belém, PA, Brasil \\ ${ }^{2}$ Universidade Estadual de Campinas, Faculdade de Engenharia Química, Campinas, SP, Brasil \\ ${ }^{3}$ Universidade Federal do Pará, Faculdade de Ciências Exatas e Tecnologia, Abaetetuba, PA, Brasil
}

\begin{abstract}
Resumo
Este trabalho teve por objetivo a síntese e caracterização do material hidrotalcita-hidroxiapatita (HTHAp) dopado com três diferentes proporções $(1,5$ e $15 \%$ p/p) de nanotubos de carbono (NTC) a fim de avaliar seu potencial como catalisador heterogêneo na reação de metanólise do óleo de soja. A síntese do material HTHAp foi realizada pelo método de coprecipitação (10 $\leq$ pH $\leq 11)$ com homogeneização ultrassônica e tratamento hidrotérmico a $80^{\circ} \mathrm{C}$. DRX, MEV/EDS, FT-IR, Raman, fisissorção de $\mathrm{N}_{2}$ e TG/ DTA foram as técnicas de caracterização utilizadas. A amostra HTHAp1NTC, dopada a $1 \%$ p/p, foi testada como catalisador em duas condições de temperatura $\left(180\right.$ e $\left.240{ }^{\circ} \mathrm{C}\right), 4$ h de tempo de reação, carga de 2,5\% de catalisador e razão álcool:óleo de $12: 1$. A dopagem contribuiu para melhorar propriedades estruturais, morfológicas e a estabilidade térmica do material. Os resultados de rendimento obtidos de $35,2 \%\left(180^{\circ} \mathrm{C}\right)$ e $40,5 \%\left(240{ }^{\circ} \mathrm{C}\right)$ qualificam o material HTHAp dopado com NTC como catalisador em potencial na reação de transesterificação.
\end{abstract}

Palavras-chave: NTC, catálise heterogênea, biodiesel, RMN ${ }^{1} \mathrm{H}$.

Abstract

The aim of this study was to synthesize and characterize hydrotalcite-hydroxyapatite (HTHAp) material doped with three different proportions $(1,5$ and $15 \% \mathrm{w} / \mathrm{w})$ of carbon nanotubes (NTC) in order to evaluate its potential as a heterogeneous catalyst in the soybean oil methanolysis reaction. The synthesis of the HTHAp material was performed by the co-precipitation method (10 $\leq$ pH $\leq$ 11) with ultrasonic homogenization and hydrothermal treatment at $80{ }^{\circ} \mathrm{C} . \mathrm{XRD}, \mathrm{SEM} / \mathrm{EDS}, \mathrm{FT}-\mathrm{IR}$, Raman, $\mathrm{N}_{2}$ physisorption and TG/DTA were the characterization techniques performed. The sample HTHAp1NTC, doped at $1 \%$ w/w, was tested as a catalyst under two temperature conditions $\left(180\right.$ and $\left.240^{\circ} \mathrm{C}\right), 4 \mathrm{~h}$ reaction time, $2.5 \%$ catalyst loading and alcohol:oil ratio of $12: 1$. Doping contributed to improve structural, morphological and thermal stability properties of HTHAp material. The yield results achieved $35.2 \%\left(180{ }^{\circ} \mathrm{C}\right)$ and $40.5 \%\left(240{ }^{\circ} \mathrm{C}\right)$ qualifying the HTHAp material doped with CNT as a potential catalyst in the transesterification reaction.

Keywords: CNT, heterogeneous catalysis, biodiesel, ${ }^{1} \mathrm{H} N M R$.

\section{INTRODUÇÃO}

$\mathrm{O}$ crescimento industrial bem como o desenvolvimento de novas tecnologias implica na necessidade de se produzir materiais de maior qualidade e desempenho funcional a partir de melhores condições de controle dos processos de fabricação dos mesmos. Dessa forma, o grande desafio no desenvolvimento de um novo material é o estabelecimento de condições de síntese ideais para a sua produção. Materiais como hidrotalcita (HT) e hidroxiapatita (HAp) são conhecidos por seus empregos como adsorventes no

*beth@ufpa.br tratamento de efluentes e como catalisadores heterogêneos e suporte para catalisadores em diversas áreas, tais como farmacêutica, médica, química, entre outras [1-3]. Catalisadores heterogêneos são amplamente aplicados industrialmente devido às vantagens que oferecem aos processos químicos, dentre elas a maior seletividade e melhor eficiência de separação catalisador/mistura reacional, o que proporciona uma redução de etapas e consequente eliminação de resíduos [4]. Um típico processo no qual essas vantagens são evidentes é a produção de biodiesel. A mudança da catálise homogênea para a heterogênea é uma oportunidade em beneficiar a produção de biodiesel em aspectos econômicos e ambientais [5]. Diversos catalisadores heterogêneos têm 
sido estudados nos últimos anos na produção de biodiesel, entre eles zeólitas, hidrotalcitas, óxidos e $\gamma$-alumina, os quais apresentam como característica mais relevante elevada área superficial [6]. Estima-se que a utilização de catalisadores heterogêneos na indústria do biodiesel reduza os custos operacionais em US\$ 59 por tonelada em comparação aos catalisadores homogêneos $[7,8]$.

A hidrotalcita (HT), de fórmula química $\mathrm{Mg}_{6} \mathrm{Al}_{2}(\mathrm{OH})_{16}\left(\mathrm{CO}_{3}\right) \cdot 4 \mathrm{H}_{2} \mathrm{O}$, pertence à classe das argilas aniônicas e é um eficiente catalisador heterogêneo na produção de biodiesel devido à sua acentuada basicidade, elevada área superficial e volume de poro, o que a torna adequada à reação de transesterificação $[9,10]$. Por outro lado, a hidroxiapatita (HAp), $\mathrm{Ca}_{5}\left(\mathrm{PO}_{4}\right)_{3}(\mathrm{OH})$, é um hidroxifosfato de cálcio amplamente utilizado na área biomédica como enxerto de tecidos duros, e industrialmente como biosensor, adsorvente e recentemente como catalisador heterogêneo [11]. Poucos trabalhos empregando a HAp na reação de transesterificação foram publicados até o momento [12,13]. A pesquisa no campo da ciência dos materiais visa estabelecer combinações e modificações que possibilitem uma vasta aplicação de novos materiais em diferentes áreas do conhecimento. Recentemente, a síntese de um material composto pelas fases hidrotalcita e hidroxiapatita (HTHAp) foi proposta e seu desempenho avaliado na transesterificação de óleo de soja e no tratamento de água [14-16]. Ademais, uma das formas mais recentes de reforço e funcionalização de matrizes inorgânicas é a utilização de nanotubos de carbono (NTC), que consistem em uma estrutura tubular formada a partir de folhas de grafeno que apresentam alta versatilidade em termos de propriedades mecânicas, elétricas e térmicas, além possibilitar um bom incremento nas propriedades estruturais de uma gama de materiais [17-19]. Na área de catálise heterogênea os NTC são predominantemente empregados como suportes para pequenas partículas de catalisador, resultando em materiais com elevada dispersão e área superficial específica, o que afeta positivamente parâmetros como atividade catalítica e seletividade [17, 20-22]. Nesse contexto, o presente trabalho se propõe a sintetizar e caracterizar o material tipo hidrotalcitahidroxiapatita (HTHAp), dopado com nanotubos de carbono, em três diferentes proporções, e testá-lo como catalisador heterogêneo na reação de transesterificação para produção de biodiesel.

\section{EXPERIMENTAL}

Sintese dos catalisadores: a síntese do material HTHAp e das amostras dopadas com NTC foi realizada por meio de coprecipitação e pH na faixa entre 10 e 11, de acordo com metodologia proposta pelo grupo de pesquisa [23]. O preparo da HT consistiu no gotejamento lento de uma solução de $\mathrm{Mg}\left(\mathrm{NO}_{3}\right)_{2} \cdot 6 \mathrm{H}_{2} \mathrm{O} 0,24$ mol.L-1 em uma solução de $\mathrm{NaOH}-$ $\mathrm{Al}$, preparada por meio da dissolução do $\mathrm{Al}^{0}{ }_{(\mathrm{s})}$ com solução de NaOH 3 mol.L $L^{-1}$, na proporção da HT estequiométrica. A síntese da HAp envolveu o gotejamento lento de uma solução de $\mathrm{H}_{3} \mathrm{PO}_{4} \quad 0,3$ mol. $\mathrm{L}^{-1}$ em uma solução de $\mathrm{Ca}(\mathrm{OH})_{2}$ 0,5 mol. $L^{-1}$, também em proporção estequiométrica. Todos os reagentes foram adquiridos da Sigma-Aldrich com pureza de $95 \%$. Os NTC foram sintetizados pelo método de corrente elétrica de curto-circuito em atmosfera inerte descritos na Patente US8808635 B2 com comprimento de $225 \mathrm{~nm}$ e diâmetro na faixa entre 0,9 e $1,2 \mathrm{~nm}$. As soluções de partida da HT e HAp foram submetidas separadamente à homogeneização ultrassônica, misturadas, acrescidas de diferentes quantidades de NTC nas proporções 1,5 e $15 \% \mathrm{p} / \mathrm{p}$ e levadas a tratamento hidrotérmico a $80{ }^{\circ} \mathrm{C} / 24 \mathrm{~h}$. Posteriormente, os precipitados foram lavados com água deionizada até $\mathrm{pH} 7$, filtrados e secos em estufa por $80^{\circ} \mathrm{C} / 24$ $\mathrm{h}$. As quatro amostras sintetizadas foram denominadas como HTHAp, HTHAp1NTC, HTHAp5NTC e HTHAp15NTC, de acordo com a proporção em massa de NTC presente na matriz do material.

Caracterização dos catalisadores: os materiais foram caracterizados por diversas técnicas: difração de raios X (DRX): PANalytical X'Pert-Pro PW 3050, CuK $\alpha$ $(\lambda=1,5406 \AA), 20 \mathrm{~s}, 30 \mathrm{~mA}, 40 \mathrm{kV}$; microscopia eletrônica de varredura/ espectrometria de energia dispersiva de raios X (MEV/EDS): Zeiss LEO 1430, metalização com Au em $2 \mathrm{~min}, 90 \mu \mathrm{A}, 10 \mathrm{kV}, 15 \mathrm{~mm}$; espectroscopia do infravermelho (FT-IR): Shimadzu IRPrestige21, $400-4000 \mathrm{~cm}^{-1}$, resolução de $4 \mathrm{~cm}^{-1}, 16$ varreduras, amostra: $\mathrm{KBr}=1: 99, \quad 1,8$; espectroscopia Raman (RS): Horiba IHR320 e Olympus bx41, câmara CCD Synapse, laser $532 \mathrm{~nm}$ (verde), fenda $200 \mu \mathrm{m}, 10 \mathrm{x}, 300$ s; fisissorção de $\mathrm{N}_{2}$ e modelagem BET: Quantachrome Instruments, pré-tratamento a $180{ }^{\circ} \mathrm{C} / 2 \mathrm{~h}$; análise termogravimétrica e termodiferencial (TG/DTA): Shimadzu DTG-60HA, 25 a $1000{ }^{\circ} \mathrm{C}, 5^{\circ} \mathrm{C} \cdot \mathrm{min}^{-1}, 200 \mathrm{~mL} \cdot \mathrm{min}^{-1} \mathrm{de} \mathrm{N}_{2}$.

Atividade catalítica: a amostra HTHAp1NTC foi avaliada como catalisador na reação de transesterificação por rota metílica. Um reator Parr ${ }^{\circledR} 4871$ foi carregado com óleo de soja Siol ${ }^{\circledR}$ e metanol P.A. $(99,9 \%)$ nas seguintes condições reacionais: temperatura de reação $\left(180\right.$ e $\left.240{ }^{\circ} \mathrm{C}\right)$, razão metanol:óleo $(12: 1)$, carga de catalisador $(2,5 \% \mathrm{p} / \mathrm{p}$ em relação a massa de óleo) e agitação constante de 700 rpm [15]. Transcorrido o tempo de reação, o produto foi filtrado a vácuo a fim de separar o catalisador sólido. Em seguida, a fase orgânica contendo os ésteres metílicos foi separada do glicerol e metanol (não reagido) por decantação, lavada com solução supersaturada de $\mathrm{NaCl}$ para remoção da glicerina remanescente e seca com $\mathrm{Na}_{2} \mathrm{SO}_{4}$ anidro.

Caracterização do biodiesel por $R M N{ }^{1} H$ : a fase orgânica foi caracterizada em um espectrômetro Varian Mercury-300, $\mathrm{CDCl}_{3}$, campo magnético $11,7 \mathrm{~T}(300$ $\mathrm{MHz}), 45^{\circ}$ pulsos, $13 \mathrm{~s}, 4120 \mathrm{~Hz}$, resolução de $0,3 \mathrm{~Hz}$ e 16 varreduras. A taxa de conversão de triglicerídeos a ésteres metílicos foi determinada por meio da equação $\mathrm{R}=100 .\left(2 \mathrm{I}_{3,7 \mathrm{ppm}} / 3 \mathrm{I}_{2,2-2,4 \mathrm{ppm}}\right)$, onde $\mathrm{I}_{3,7 \mathrm{ppm}}$ é a integral da área correspondente aos sinais dos hidrogênios metoxílicos dos ésteres ( $\left.\mathrm{CH}_{3} \mathrm{OCO}-\right)$ e $\mathrm{I}_{2,2-2,4 \mathrm{ppm}}$ é a integral da área atribuída aos prótons metilênicos $\alpha$-carbonílicos $\left(-\mathrm{OCOCH}_{2}-\right)$ [24]. $\mathrm{O}$ valor da concentração de glicerina livre no produto é dado por $\mathrm{GL}(\% \mathrm{p} / \mathrm{p})=\left(\mathrm{I}_{5,3-5,2 \mathrm{ppm}}-\mathrm{r}\right) /(\mathrm{r} . \mathrm{W})$, onde $\mathrm{I}_{5,3-5,2 \mathrm{ppm}}$ é a integral dos sinais dos prótons na posição sn-2 do glicerol, 
$\mathrm{r}=\mathrm{I}_{4,40-4,05 \mathrm{ppm}} / 2, \mathrm{I}_{4,40-4,05 \mathrm{ppm}}$ é a área correspondente aos sinais dos prótons nas posições sn-1 e sn-3 ( $\delta$ 4,05-4,40 ppm) e W é a massa molecular da glicerina (92 g/mol) [25].

\section{RESULTADOS E DISCUSSÃO}

$D R X$ : o difratograma da amostra HTHAp (hidrotalcitahidroxiapatita) apresentou reflexões características das fases

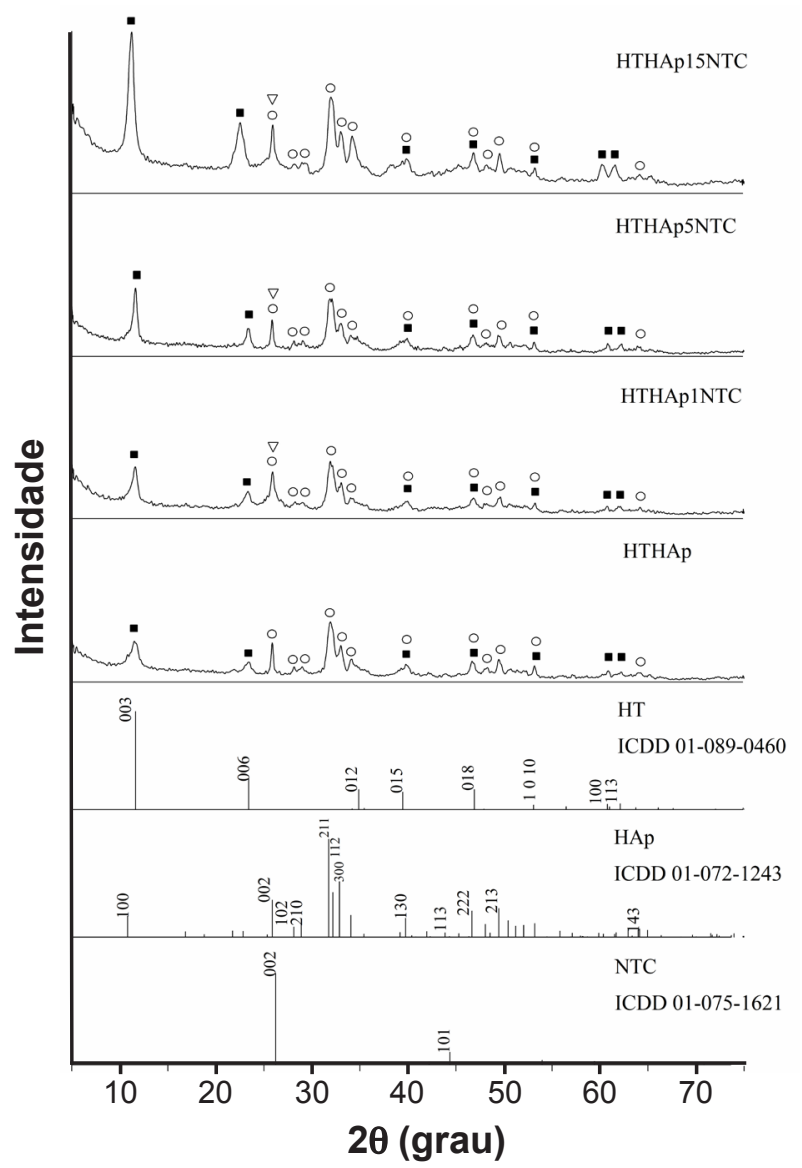

Figura 1: Difratogramas de raios $\mathrm{X}$ dos materiais sintetizados e minerais de referência. Fases: HT (ロ), HAp (O) e NTC ( $\nabla)$.

[Figure 1: XRD patterns of synthesized materials and reference minerals. Phases: HT (ם), HAp (O), and NTC ( $\nabla)$.] que a compõe: HT [(003), (006), (012), (018), (015), (113), (110)] e HAp [(211), (300), (112), (002), (213), (222)]. Os materiais dopados com NTC (HTHAp1NTC, HTHAp5NTC e HTHAp15NTC) apresentaram um pico adicional referente ao plano (002) do NTC, indicando a eficiência da rota de síntese uma vez que não houve cristalização de fases acessórias (Fig. 1). Todas as amostras exibiram boa cristalinidade, evidenciada pela intensidade e definição dos picos. O caráter lamelar da fase HT foi comprovado pela presença de um pico intenso próximo a $2 \theta=10^{\circ}$, relativo ao plano (003). Esse pico mostrou-se mais intenso nos materiais dopados com NTC, o que aponta para uma melhora na cristalização da estrutura lamelar com a incorporação de NTC na matriz do material. Em relação à fase HAp, todas as amostras, dopadas ou não, apresentaram os picos principais com deslocamentos para esquerda em relação ao mineral de referência, exceto pelo plano (100) que não foi observado nas amostras dopadas. O mesmo comportamento foi reportado na literatura [14], provavelmente devido à superposição pelo plano (003) da fase HT que é muito mais intenso. A introdução de NTC na matriz do material, ainda que tenha contribuído para melhorar a cristalinidade, aparentemente não afetou os parâmetros de cela unitária, os quais apresentaram valores em concordância com os minerais de referência tanto da fase HAp quanto da fase HT (Tabela I).

$M E V / E D S$ : os cristais formados em todas as amostras (dopadas ou não) apresentaram dimensão variando entre 1 e $5 \mu \mathrm{m}$ (Fig. 2). A análise de EDS da amostra HTHAp indicou a presença dos elementos constituintes das fases HT e HAp. A razão catiônica $\mathrm{Mg} / \mathrm{Al}$ observada na amostra variou entre 2,06 e 2,17, enquanto que o valor estequiométrico é 3. A razão x $[\mathrm{Al} /(\mathrm{Al}+\mathrm{Mg})]$ apresentou um valor constante de 0,32 , próximo ao valor da HT estequiométrica $(0,33)$. Quanto às razões $\mathrm{Ca} / \mathrm{P}$, as mesmas variaram entre 1,29 e 1,43, valores bem inferiores ao valor estequiométrico de 1,67 (Tabela II). Essas variações eram esperadas uma vez que o ambiente de síntese utilizado foi diferente daquele usualmente empregado utilizando reagentes de alta pureza. Além disso, possíveis substituições iônicas entre os precursores podem ter ocorrido e refletiram na composição final do material. Os valores apresentados na Tabela II foram obtidos a partir da análise de

Tabela I - Parâmetros de cela unitária do material HTHAp e amostras dopadas com NTC.

[Table I - Unit cell parameters of HTHAp material and samples doped with NTC.]

\begin{tabular}{ccccccc}
\hline \multirow{2}{*}{ Amostra } & \multicolumn{3}{c}{ Fase HT } & \multicolumn{3}{c}{ Fase HAp } \\
& $\mathrm{a}=\mathrm{b}(\AA)$ & $\mathrm{c}(\AA)$ & $\mathrm{V}\left(\AA^{3}\right)$ & $\mathrm{a}=\mathrm{b}(\AA)$ & $\mathrm{c}(\AA)$ & $\mathrm{V}\left(\AA^{3}\right)$ \\
\hline HTHAp & $3,03 \pm 0,02$ & $22,4 \pm 0,1$ & $177,9 \pm 2,1$ & $9,44 \pm 0,01$ & $6,90 \pm 0,01$ & $531,9 \pm 1,5$ \\
HTHAp1NTC & $3,04 \pm 0,01$ & $22,6 \pm 0,1$ & $180,6 \pm 1,6$ & $9,43 \pm 0,02$ & $6,91 \pm 0,01$ & $531,7 \pm 2,2$ \\
HTHAp5NTC & $3,02 \pm 0,02$ & $22,3 \pm 0,2$ & $176,1 \pm 2,6$ & $9,44 \pm 0,03$ & $6,92 \pm 0,03$ & $533,4 \pm 4,1$ \\
HTHAp15NTC $^{3}$ & $3,04 \pm 0,01$ & $22,5 \pm 0,2$ & $179,5 \pm 1,9$ & $9,39 \pm 0,02$ & $6,86 \pm 0,01$ & $523,7 \pm 2,3$ \\
HT $^{\mathrm{a}}$ & 3,046 & 22,772 & 182,97 & - & - & - \\
HAp $^{\mathrm{b}}$ & - & - & - & 9,432 & 6,881 & 530,123 \\
HT-HAp [15] $^{2}$ & $3,038 \pm 0,010$ & $22,67 \pm 0,20$ & $181,1 \pm 1,9$ & $9,375 \pm 0,034$ & $6,874 \pm 0,026$ & $523,2 \pm 4,3$ \\
\hline Notas: $^{a}-$ ICDD ficha PDF 01-089-0460; ${ }^{b}-$ ICDD ficha PDF $01-072-1243$. &
\end{tabular}



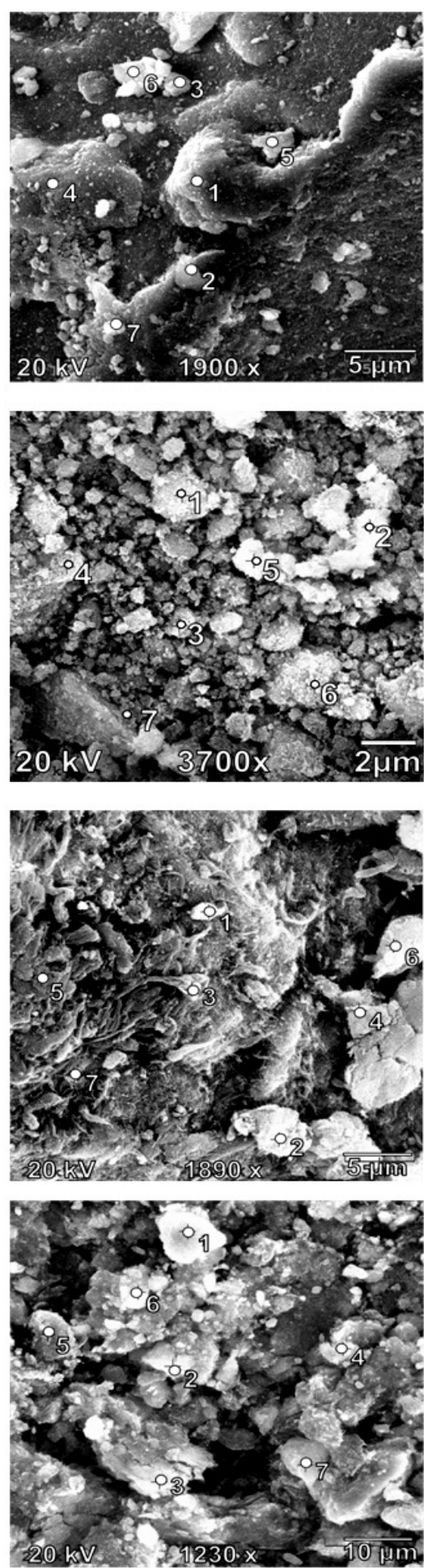

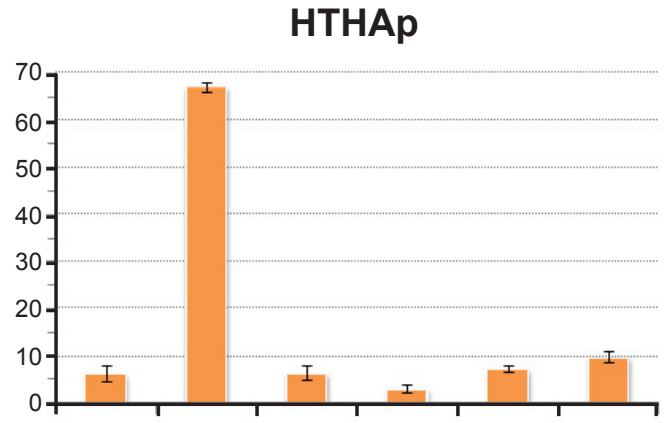

HTHAp1NTC

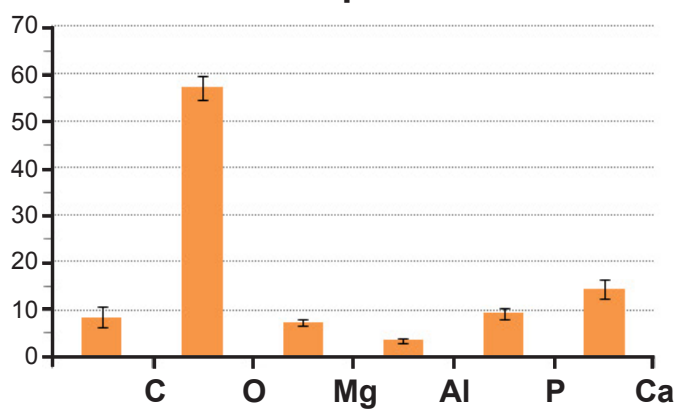

HTHAp5NTC

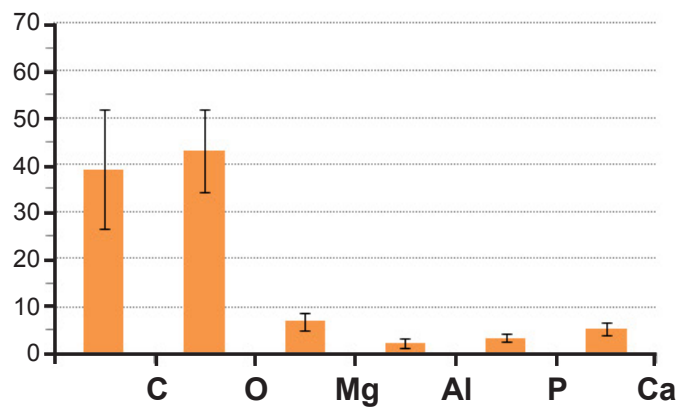

HTHAp15NTC

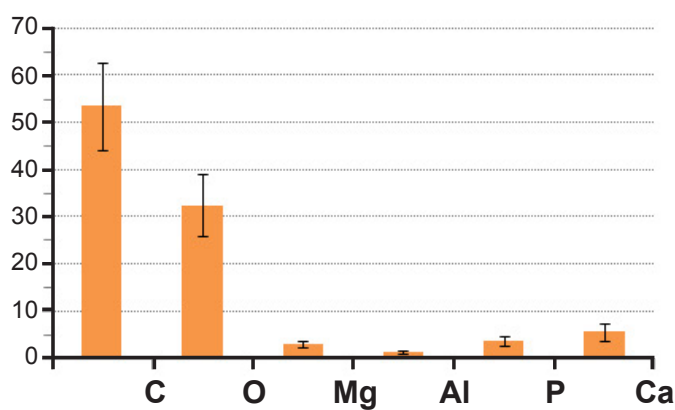

Figura 2: Micrografias e análises elementares semiquantitativas (\%at) das amostras sintetizadas.

[Figure 2: Micrographs and semi-quantitative elemental analysis (at\%) of the synthesized samples.]

7 pontos de cada amostra. Ao se introduzir $1 \%$ p/p de NTC na matriz do material, o sistema pareceu não sofrer perturbações. Entretanto, 5 e $15 \%$ p/p provocaram variações significativas em todas as razões catiônicas mensuradas indicando uma cristalização heterogênea dos materiais dopados.

FT-IR: as amostras sintetizadas apresentaram as bandas características dos grupos inorgânicos presentes nos minerais de referência, fase $\mathrm{HT}\left(\mathrm{Mg}, \mathrm{Al}-\mathrm{OH}, \mathrm{CO}_{3}{ }^{2-}, \mathrm{OH}^{-}\right)$, e fase $\mathrm{HAp}\left(\mathrm{PO}_{4}^{3-}, \mathrm{OH}^{-}\right)$além de moléculas de $\mathrm{H}_{2} \mathrm{O}$ adsorvidas (Fig. 3 e Tabela III). As amostras dopadas com NTC apresentaram redução na intensidade das bandas referentes às fases HT e HAp com o aumento do teor de nanotubos 
Tabela II - Razões elementares das fases HT e HAp.

[Table II - Elementary ratios of HT and HAp phases.]

\begin{tabular}{cccc}
\hline Amostra & $\mathrm{Mg} / \mathrm{Al}$ & $\mathrm{x}[\mathrm{Al} /(\mathrm{Mg}+\mathrm{Al})]$ & $\mathrm{Ca} / \mathrm{P}$ \\
\hline HTHAp & $2,06-2,17$ & $\sim 0,32$ & $1,29-1,43$ \\
HTHAp1NTC & $1,99-2,12$ & $\sim 0,33$ & $1,47-1,64$ \\
HTHAp5NTC & $1,86-4,07$ & $0,20-0,35$ & $1,38-1,74$ \\
HTHAp15NTC & $2,35-2,87$ & $0,26-0,30$ & $1,40-1,58$ \\
\hline
\end{tabular}

na matriz dos materiais sintetizados. Todas as amostras exibiram bandas intensas na região em torno de $3550 \mathrm{~cm}^{-1}$ associadas às vibrações de estiramento da ligação $\mathrm{O}-\mathrm{H}$ das hidroxilas presentes nas camadas da fase HT e aos grupos $\mathrm{OH}^{-}$da fase HAp. As ondulações registradas em torno de $3000 \mathrm{~cm}^{-1}$ foram atribuídas às ligações de hidrogênio entre as moléculas de $\mathrm{H}_{2} \mathrm{O}$ e os íons $\mathrm{CO}_{3}^{2-}$. A intensidade desta ondulação é diretamente proporcional ao grau de cristalização da fase HT [26]. Bandas na região de baixos números de onda foram atribuídas à deformação angular das moléculas de $\mathrm{H}_{2} \mathrm{O}$ em torno de $1600 \mathrm{~cm}^{-1}$ relativas à fase $\mathrm{HT}$, e a $\mathrm{OH}^{-}$da fase HAp em aproximadamente $630 \mathrm{~cm}^{-1}$. As bandas associadas aos modos vibracionais (3) do íon $\mathrm{CO}_{3}{ }^{2-}$ da fase $\mathrm{HT}$ foram observadas em todas as amostras. A banda intensa e larga em torno de $1370 \mathrm{~cm}^{-1}$ foi atribuída ao modo $v_{3}$ da vibração de estiramento antissimétrico; bandas menos intensas localizadas nas regiões de 870 e $650 \mathrm{~cm}^{-1}$ foram atribuídas aos modos $v_{2}$ e $v_{4}$ que corresponderam respectivamente às vibrações de flexão no plano e fora do plano. O modo $v_{1}$ é inativo na região do infravermelho [27]. As amostras sintetizadas apresentaram desvios no modo $v_{4}$ devido à sobreposição da intensa banda relacionada ao íon $\mathrm{PO}_{4}^{3-}$ presente na fase HAp. Os modos vibracionais $v_{2}, v_{3} \mathrm{e}$ $v_{4}$ corresponderam a possíveis ambientes diferentes do íon $\mathrm{CO}_{3}^{2--}$ 1) íon $\mathrm{CO}_{3}{ }^{2-}$ livre; 2) íon $\mathrm{CO}_{3}{ }^{2-}$ ligado à molécula de $\mathrm{H}_{2} \mathrm{O}$; e 3) íon $\mathrm{CO}_{3}{ }^{2-}$ ligado a um grupo $\mathrm{OH}^{-}$na superfície da camada da HT [28]. Registraram-se bandas relativas às vibrações das ligações metal-oxigênio da fase HT em todas as amostras. A banda aguda na região de $760 \mathrm{~cm}^{-1}$ foi associada à vibração de translação da ligação Al-O. A banda em $550 \mathrm{~cm}^{-1}$ foi atribuída ao modo translacional do grupo $\mathrm{OH}^{-}$influenciado pelo cátion $\mathrm{Al}^{3+}$ (translação $\mathrm{Mg}, \mathrm{Al}-$ $\mathrm{OH}$ ) [29]. A banda na região em torno de $2340 \mathrm{~cm}^{-1}$ pode ser atribuída à deformação angular da ligação $(\delta, \mathrm{O}-\mathrm{C}-\mathrm{O})$, que está relacionada ao $\mathrm{CO}_{2}$ presente em inclusões fluidas do mineral. Observaram-se bandas referentes aos modos vibracionais (3) do íon $\mathrm{PO}_{4}^{3-}$, oriundo da fase $\mathrm{HAp}$, em todas as amostras. As bandas intensas na região de 1092 e 1036 $\mathrm{cm}^{-1}$ são características do modo vibracional $v_{3}$, atribuído a vibração de estiramento antissimétrico da ligação P-O. A banda fraca em torno de $961 \mathrm{~cm}^{-1}$ foi atribuída ao modo vibracional $v_{1}$ que corresponde ao estiramento simétrico

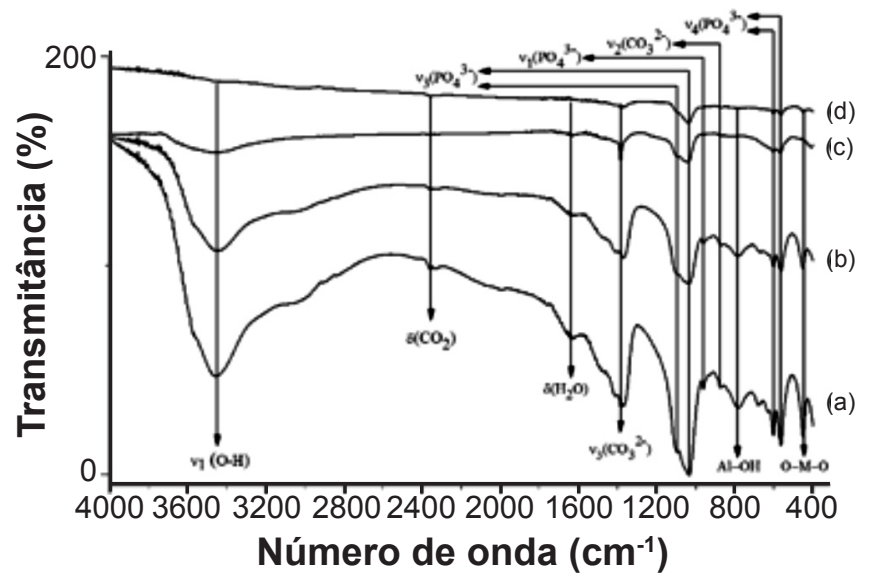

Figura 3: Espectros de FT-IR das amostras: (a) HTHAp; (b) HTHAp1NTC; (c) HTHAp5NTC; e (d) HTHAp15NTC.

[Figure 3: FT-IR spectra of samples: (a) HTHAp; (b) HTHApINTC; (c) HTHAp5NTC; and (d) HTHAp15NTC.]

Tabela III - Bandas de absorção $\left(\mathrm{cm}^{-1}\right)$ das amostras sintetizadas e minerais de referência.

[Table III - Absorption bands $\left(\mathrm{cm}^{-1}\right)$ of synthesized samples and reference minerals.]

\begin{tabular}{cccccccc}
\hline Banda & HTHAp & HTHAp1NTC & HTHAp5NTC & HTHAp15NTC & HT [32] & Hap [33] & HT-Hap [15] \\
\hline$v_{1}(\mathrm{O}-\mathrm{H})$ & 3458 & 3451 & 3455 & 3447 & 3570 & - & 3446 \\
$\delta\left(\mathrm{H}_{2} \mathrm{O}\right)$ & 1632 & 1632 & 1630 & 1629 & 1640 & - & 1625 \\
$v_{3}\left(\mathrm{CO}_{3}{ }^{2-}\right)$ & 1383 & 1369 & 1385 & 1371 & 1370 & 1400 & 1370 \\
$v_{3}\left(\mathrm{PO}_{4}{ }^{3-}\right)$ & 1093 & 1092 & 1092 & 1091 & - & 1095 & 1087 \\
$v_{3}\left(\mathrm{PO}_{4}{ }^{3-}\right)$ & 1032 & 1034 & 1040 & 1040 & - & 1036 & 1032 \\
$v_{1}\left(\mathrm{PO}_{4}{ }^{3-}\right)$ & 962 & 961 & 962 & 960 & - & 963 & 952 \\
$v_{2}\left(\mathrm{CO}_{3}{ }^{2-}\right)$ & 872 & 874 & 874 & 872 & 870 & 870 & 852 \\
$v_{4}\left(\mathrm{CO}_{3}{ }^{2-}\right)$ & 681 & 669 & - & 667 & 650 & - & 672 \\
$v_{4}\left(\mathrm{PO}_{4}{ }^{3-}\right)$ & 602 & 602 & 602 & 604 & - & 600 & 600 \\
$v_{4}\left(\mathrm{PO}{ }^{3-}\right)$ & 563 & 563 & 565 & 563 & - & 568 & 560 \\
$\mathrm{Al}-\mathrm{O}$ & 779 & 781 & 810 & 777 & 760 & - & - \\
$\mathrm{Mg}, \mathrm{Al}-$ & 563 & 563 & 565 & 563 & 554 & - & 560 \\
$\mathrm{OH}$ & & & & & & & \\
\hline
\end{tabular}




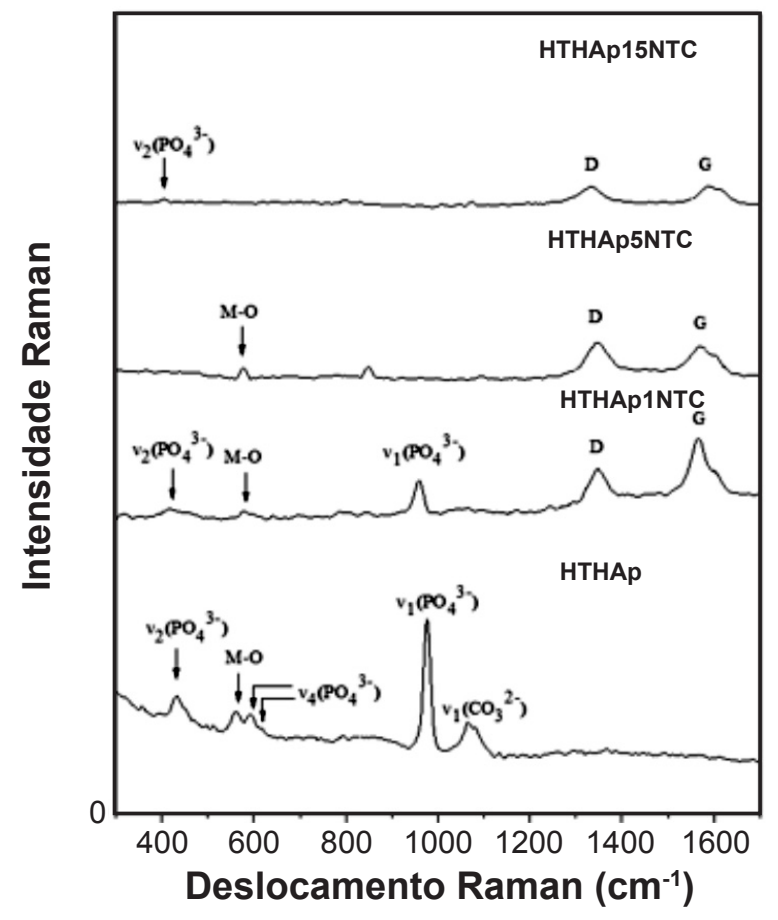

Figura 4: Espectros Raman das amostras sintetizadas.

[Figure 4: Raman spectra of synthesized samples.]

da ligação P-O. As bandas agudas entre 550 e $610 \mathrm{~cm}^{-1}$ foram associadas ao modo vibracional $v_{4}$, atribuído à flexão da ligação O-P-O. Essas bandas são características de estruturas moleculares dos poliedros de $\mathrm{PO}_{4}^{3-}$ na estrutura da HAp [30]. As bandas mais intensas dos NTC, localizadas em 1580 e $868 \mathrm{~cm}^{-1}$, atribuídas aos modos vibracionais ativos característicos dos NTC de parede simples, não foram observadas nas amostras dopadas com NTC. A dificuldade na detecção foi atribuída ao fato que os NTC não suportam um momento dipolar estático [31].

Espectroscopia Raman: a amostra HTHAp apresentou as bandas características dos grupos inorgânicos das fases $\mathrm{HT}\left(\mathrm{Mg}, \mathrm{Al}-\mathrm{OH}, \mathrm{CO}_{3}{ }^{2-}\right)$ e $\mathrm{HAp}\left(\mathrm{PO}_{4}{ }^{3-}\right)$. As amostras dopadas com NTC também exibiram bandas relativas às fases HT e HAp, além das bandas D e G dos NTC. Novamente foi observada uma redução da intensidade das bandas com o aumento do teor de NTC na matriz dos materiais (Fig. 4 e Tabela IV), em concordância com os espectros de FT-IR. A presença do íon $\mathrm{CO}_{3}{ }^{2-}$ da fase HT na amostra HTHAp foi registrada na região de 1060 e $680 \mathrm{~cm}^{-1}$, correspondentes aos modos vibracionais $v_{1}$ e $v_{4}$ referentes ao estiramento antissimétrico e flexão angular da ligação (O-C-O) do íon $\mathrm{CO}_{3}^{2-}$ livre [29]. Essas bandas não foram detectadas nas amostras dopadas. A amostra HTHAp exibiu uma banda na região de $560 \mathrm{~cm}^{-1}$ atribuída a translação do grupo $\mathrm{OH}^{-}$ associado a metais $\mathrm{Mg}$ ou Al da fase HT. A inserção de NTC parece limitar esse movimento no material visto que nas amostras dopadas com 1 e $5 \%$ ocorreu um deslocamento tênue para regiões de alta frequência enquanto que na dopagem de $15 \%$ não houve detecção. A amostra HTHAp apresentou bandas do íon $\mathrm{PO}_{4}^{3-}$ nas regiões de 960, 590610 e $430 \mathrm{~cm}^{-1}$ associadas aos modos $v_{1}, v_{4}$ e $v_{2}$, atribuídos às vibrações de estiramento simétrico da ligação $(\mathrm{P}-\mathrm{O})$ do tetraedro de $\mathrm{PO}_{4}^{3-}$, flexão angular triplamente degenerada da unidade (O-P-O) e flexão angular duplamente degenerada da unidade (O-P-O), respectivamente [34]. As amostras dopadas com NTC apresentaram modos vibracionais em regiões de alta frequência, nos valores em torno de 1340 e $1580 \mathrm{~cm}^{-1}$, atribuídos às bandas $\mathrm{D} \mathrm{e} \mathrm{G}$, sendo a banda $\mathrm{D}$ atribuída à presença de carbono amorfo, enquanto a banda $\mathrm{G}$ é associada à presença de tubos de carbono grafite ordenados [36]. Essas bandas do NTC se sobressaíram às demais, o que pode ser observado na mudança nos espectros com o aumento da proporção de dopagem.

Fisissorção de $N_{2}$ : a amostra HTHAp apresentou valores de área superficial específica $\left(\mathrm{S}_{\mathrm{BET}}\right)$, volume de poro $\left(\mathrm{V}_{\mathrm{P}}\right)$ e diâmetro médio de poro $\left(\mathrm{D}_{\mathrm{P}}\right)$ próximos ao mineral de referência descrito na literatura. A introdução de NTC na matriz das amostras melhorou significativamente suas propriedades morfológicas, aumentando todos os parâmetros supracitados (Tabela V). Em relação à área superficial específica, verificou-se um aumento linear com a quantidade de NTC dopado. Essa característica é

Tabela IV - Modos vibracionais Raman $\left(\mathrm{cm}^{-1}\right)$ das amostras HTHAp, das dopadas com NTC e minerais de referência. [Table IV - Raman vibrational modes $\left(\mathrm{cm}^{-1}\right)$ of HTHAp, NTC doped samples, and reference minerals.]

\begin{tabular}{|c|c|c|c|c|c|c|c|c|}
\hline Banda & HTHAp & HTHAp1NTC & HTHAp5NTC & HTHAp15NTC & HT [29] & HAp [34] & NTC [35] & HT-HAp [15] \\
\hline$v_{2}\left(\mathrm{PO}_{4}^{3-}\right)$ & 434 & 418 & 439 & 404 & - & 433 & - & 439 \\
\hline $\begin{array}{c}\mathrm{Mg}, \mathrm{Al}- \\
\mathrm{OH}\end{array}$ & 562 & 576 & 578 & - & 550 & - & - & 548 \\
\hline$v_{4}\left(\mathrm{PO}_{4}^{3-}\right)$ & 594 & - & - & - & - & 594 & - & 583 \\
\hline$v_{4}\left(\mathrm{PO}_{4}^{3-}\right)$ & 614 & - & - & - & - & 610 & - & 600 \\
\hline$v_{4}\left(\mathrm{CO}_{3}^{2-}\right)$ & 693 & - & - & - & 680 & - & - & - \\
\hline$v_{1}\left(\mathrm{PO}_{4}^{3-}\right)$ & 976 & 958 & - & - & - & 961 & - & 960 \\
\hline$v_{1}\left(\mathrm{CO}_{3}{ }^{2-}\right)$ & 1064 & - & - & - & 1063 & - & - & 1065 \\
\hline Banda D & - & 1344 & 1345 & 1335 & - & & 1342 & - \\
\hline Banda $\mathrm{G}$ & - & 1566 & 1570 & 1588 & - & & 1584 & - \\
\hline
\end{tabular}


Tabela V - Propriedades morfológicas da amostra sintetizadas e valores da referência.

[Table V-Morphological properties of synthesized sample and literature values.]

\begin{tabular}{cccc}
\hline Amostra & $\mathrm{S}_{\mathrm{BET}}\left(\mathrm{m}^{2} \cdot \mathrm{g}^{-1}\right)$ & $\mathrm{V}_{\mathrm{P}}\left(\mathrm{cm}^{3} \cdot \mathrm{g}^{-1}\right)$ & $\mathrm{D}_{\mathrm{P}}(\AA)$ \\
\hline HTHAp & 82 & 0,25 & 40,0 \\
HTHAp1NTC & 88 & 0,53 & 197,5 \\
HTHAp5NTC & 112 & 0,70 & 225,7 \\
HTHAp15NTC & 146 & 0,77 & 248,2 \\
HTHAp [15] & 85 & 0,54 & 17,9 \\
NTC [32] & 577 & 1,06 & 74,0 \\
\hline
\end{tabular}

- Isoterma de adsorção $\left(\mathrm{N}_{2} 77 \mathrm{~K}\right)$

- - Isoterma de dessorção

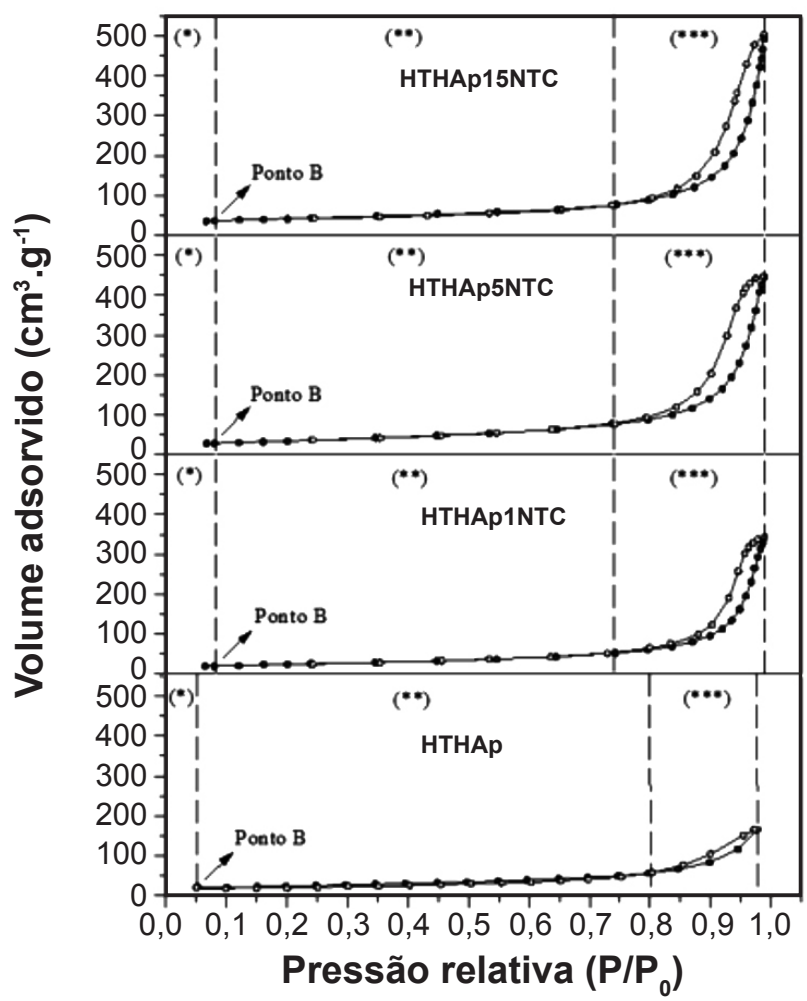

Figura 5: Isotermas de adsorção-dessorção de $\mathrm{N}_{2}$ da HTHAp e das amostras dopadas com NTC: $(*)$ monocamada; $(* *)$ multicamada; (***) condensação capilar.

[Figure 5: $N_{2}$ adsorption-desorption isotherms of HTHAp and NTC doped samples: (*) monolayer; (**) multilayer; (***) capillary condensation.]

comumente observada na literatura uma vez que o NTC evita compactação do material e induz maior dispersão das partículas de catalisador. Os valores de $\mathrm{V}_{\mathrm{P}}$ e $\mathrm{D}_{\mathrm{P}}$ do material HTHAp diferiram dos descritos na literatura e devem refletir o grau de cristalinidade e distribuição das fases HT e HAp no material. Nesse trabalho, foi verificado que a fase HAp apresentou uma melhor cristalização em relação à fase HT enquanto que o material preparado anteriormente [15] continha ambas as fases igualmente bem cristalizadas.
Tabela VI - Posição do ponto B nas isotermas de adsorção das amostras sintetizadas.

[Table VI - B point position in the adsorption isotherms of synthesized samples.]

\begin{tabular}{ccc}
\hline Amostra & $\begin{array}{c}\text { Posição do ponto } \\
\mathrm{B}\left(\mathrm{cm}^{3} \cdot \mathrm{g}^{-1}\right)\end{array}$ & $\begin{array}{c}\text { Variação em } \\
\text { relação à amostra } \\
\text { HTHAp }\end{array}$ \\
\hline HTHAp & 19,57 & - \\
HTHAp1NTC & 20,11 & $3 \%$ \\
HTHAp5NTC & 28,52 & $31 \%$ \\
HTHAp15NTC & 36,39 & $46 \%$ \\
\hline
\end{tabular}

Isotermas do tipo IV, características de materiais mesoporosos $\left(20<\mathrm{D}_{\mathrm{P}}<500 \AA\right)$, foram registradas para todas as amostras sintetizadas. A forma dessas isotermas é decorrente da cobertura da superfície do material mesoporoso, e consequente preenchimento de poros por $\mathrm{N}_{2}$. As isotermas de adsorção e dessorção com histerese do tipo H3 foram obtidas para todas as amostras, caracterizadas pela presença de aglomerados ou agregados não rígidos de partículas com forma de placas paralelas que dão origem a poros em forma de fendas ou cunhas (Fig. 5). A amostra HTHAp apresentou um comportamento diferente das amostras dopadas. $\mathrm{O}$ ponto $\mathrm{B}$ na Fig. 5 corresponde à região da isoterma onde ocorre transição da formação da monocamada de adsorbato e iniciase a multicamada [37]. A posição do ponto B foi deslocada com a introdução de NTC na matriz do material: $3 \%$ para a amostra HTHAp1NTC, 31\% para a amostra HTHAp5NTC e $46 \%$ para a amostra HTHAp15NTC (Tabela VI). Esse comportamento pode estar diretamente relacionado com as elevadas áreas superficiais dos materiais dopados.

TG/DTA: todas as amostras sintetizadas apresentaram perfis térmicos com três etapas endotérmicas de perda de massa (Fig. 6). O primeiro evento correspondeu à remoção da água adsorvida na superfície das fases HT e HAp, bem como a perda das moléculas de água interlamelar da fase HT. Em seguida, ocorreu o início dos processos de desidroxilação e descarbonatação da fase HT e, por fim, o término da remoção dos grupos $\mathrm{OH}^{-}$e $\mathrm{CO}_{3}{ }^{2-}$ remanescentes. A inserção de NTC na matriz do material favoreceu maior estabilidade térmica, elevando as temperaturas nas quais ocorreram as perdas de massa (Tabela VII). A amostra HTHAp1NTC apresentou perda de massa total inferior à amostra não dopada, o que pode ser consequência do baixo teor de NTC. O aumento da quantidade de NTC dopado aumentou a porcentagem total de perda de massa devido à maior quantidade de material decomposto no terceiro evento (decomposição de NTC). Na amostra HTHAp15NTC, a temperatura na qual o material foi completamente desidroxilado e descarbonatado passou de 478 para $766^{\circ} \mathrm{C}$. Em relação ao material HTHAp sintetizado em estudo prévio, a maior presença da fase HAp na composição do material reduziu a intensidade dos eventos de desidroxilação e descarbonatação da estrutura lamelar da HT e, consequentemente, diminuiu a porcentagem total de perda de massa. 


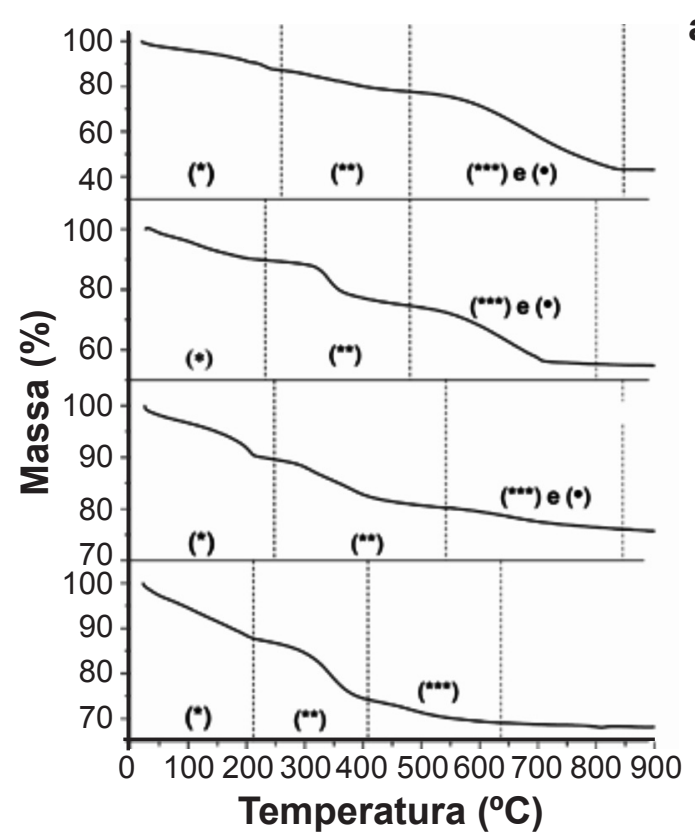

a)

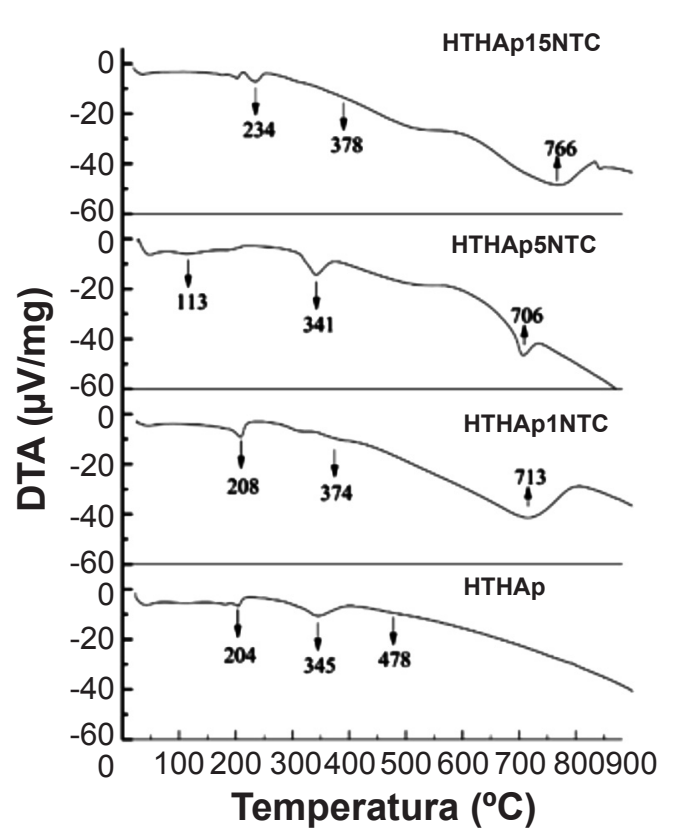

b)

Figura 6: Curvas TG/DTA das amostras HTHAp e dopadas com NTC: (*) desidratação das fases HT e HAp; (**) início da desidroxilação + descarbonatação da fase HT; $(* * *)$ término da desidroxilação + descarbonatação da fase HT; $(\bullet)$ decomposição dos NTC.

[Figure 6: TG/DTA curves of HTHAp and NTC doped samples: (*) dehydration of HT and HAp phases; (**) beginning of dehydroxylation + decarbonation of HT phase; (***) end of dehydroxylation + decarbonation of HT phase; $(\bullet)$ NTC decomposition.]

Tabela VII - Análise térmica das amostras sintetizadas e valores de referência. [Table VII - Thermal analysis of synthesized samples and reference values.]

\begin{tabular}{cccccccc}
\hline \multirow{2}{*}{ Amostra } & \multicolumn{2}{c}{ Desidratação } & \multicolumn{3}{c}{ Desidroxilação + descarbonatação + decomposição do NTC } & \multicolumn{2}{c}{ Perda total } \\
& $\% \mathrm{p} / \mathrm{p}$ & $\mathrm{T}_{\text {máx }}\left({ }^{\circ} \mathrm{C}\right)$ & $\% \mathrm{p} / \mathrm{p}$ & $\mathrm{T}_{\text {máx }}\left({ }^{\circ} \mathrm{C}\right)$ & $\% \mathrm{p} / \mathrm{p}$ & $\mathrm{T}_{\text {máx }}\left({ }^{\circ} \mathrm{C}\right)$ & $(\% \mathrm{p} / \mathrm{p})$ \\
\hline HTHAp & 11,7 & 204 & 14,0 & 345 & 4,2 & 478 & 29,9 \\
HTHAp1NTC & 9,8 & 208 & 9,2 & 374 & 4,8 & 713 & 23,8 \\
HTHAp5NTC & 10,9 & 113 & 15,8 & 341 & 19,7 & 706 & 46,4 \\
HTHAp15NTC & 10,8 & 234 & 7,9 & 378 & 30,3 & 766 & 49,0 \\
HT [38] & 17,0 & $75,220,235$ & 10,3 & 365 & 17,0 & 415 & 44,3 \\
HAp [39] & 16,0 & 250 & - & - & - & - & $22^{*}$ \\
NTC [40] & - & 80 & - & - & - & 600 & - \\
HTHAp [15] & 15,8 & 168 & 12,0 & 342 & 6,9 & 540 & 34,7 \\
\hline
\end{tabular}

Reação de transesterificação: os espectros de RMN ${ }^{1} \mathrm{H}$ foram obtidos para os produtos líquidos e purificados das reações e estão apresentados na Fig. 7. Os hidrogênios metílicos, utilizados para o cálculo do rendimento em biodiesel, são localizados no deslocamento químico de $3,7 \mathrm{ppm}$. Foi verificado que tanto a $180{ }^{\circ} \mathrm{C}$ como a 240 ${ }^{\circ} \mathrm{C}$ o material dopado HTHAp1NTC apresentou atividade catalítica (rendimentos de $35,2 \%$ e $40,5 \%$, respectivamente). $\mathrm{O}$ rendimento a ésteres metílicos $(\mathrm{R})$ e o teor de glicerina livre (GL) nas duas temperaturas estão apresentados na Tabela VIII. O rendimento a ésteres metílicos foi favorecido pelo aumento de temperatura, provável consequência da diminuição dos efeitos de transferência de massa ligados à catálise heterogênea. A detecção de sinais no deslocamento químico entre 4 e 4,4 ppm, típica de mono, di e triglicerídeos, confirmou que a reação não foi completa. Em temperatura mais branda $\left(180{ }^{\circ} \mathrm{C}\right)$, o material dopado, HTHAp1NTC, apresentou rendimento aproximadamente 50\% superior ao resultado obtido para o material não dopado, indicando melhora do desempenho catalítico com a inserção de NTC na matriz inorgânica da HTHAp. Entretanto, com o aumento de temperatura $\left(240{ }^{\circ} \mathrm{C}\right)$, a presença dos NTC apresentou um efeito negativo na atividade catalítica. Esse resultado foi coerente dado que uma das características obtidas pela dopagem com NTC é a estabilidade térmica sob as condições reacionais [22]. Acredita-se que a elevação de temperatura pode ter modificado a morfologia do material não dopado de forma que houve um aumento significativo em sua atividade 


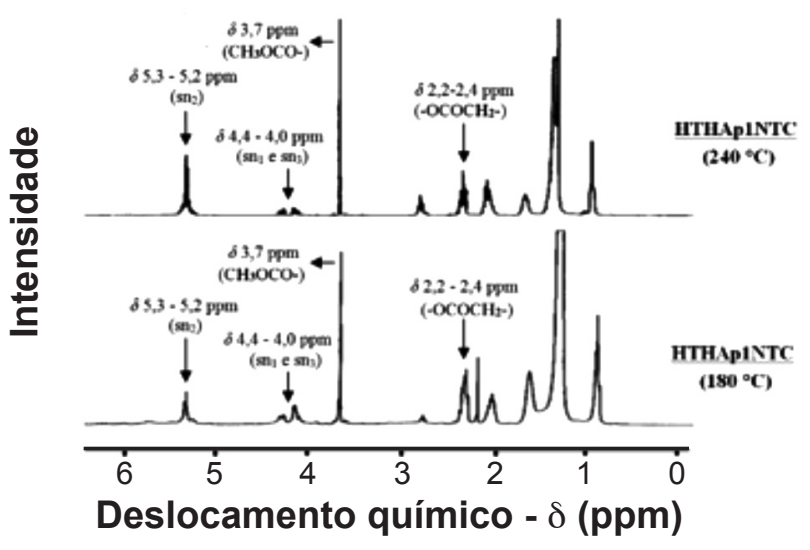

Figura 7: Espectros de $\mathrm{RMN}{ }^{1} \mathrm{H}$ do óleo de soja e dos produtos das reações.

[Figure 7: ${ }^{1} H$ NMR spectra of soybean oil and reaction products.]

Tabela VIII - Rendimento em ésteres metílicos (R) e teor de glicerina livre (GL) dos produtos de reação.

[Table VIII - Yield in methyl esters $(R)$ and free glycerin content $(G L)$ of the reaction products.]

\begin{tabular}{ccccc}
\hline & \multicolumn{2}{c}{$180{ }^{\circ} \mathrm{C}$} & \multicolumn{2}{c}{$240{ }^{\circ} \mathrm{C}$} \\
& $\mathrm{R}(\%)$ & $\mathrm{GL}(\% \mathrm{p} / \mathrm{p})$ & $\mathrm{R}(\%)$ & $\mathrm{GL}(\% \mathrm{p} / \mathrm{p})$ \\
\hline HTHAp1NTC & 35,2 & 0,059 & 40,5 & 0,152 \\
HTHAp [15] & 23,8 & 0,048 & 80,4 & - \\
\hline
\end{tabular}

catalítica. Por outro lado, a inserção de NTC na matriz do material, apesar de melhorar a estabilidade térmica, limitou a resposta do rendimento de reação à elevação de temperatura, sendo, portanto, mais eficiente em condições reacionais mais brandas.

\section{CONCLUSÕES}

A síntese do material HTHAp (hidrotalcitahidroxiapatita) foi realizada via coprecipitação $(10 \leq \mathrm{pH} \leq 11)$, homogeneização ultrassônica e tratamento hidrotérmico a $80{ }^{\circ} \mathrm{C}$, com introdução de NTC (nanotubos de carbono) em três diferentes proporções $(1,5$ e $15 \%$ p/p): HTHAp1NTC, HTHAp5NTC e HTHAp15NTC. Dados de DRX apontaram a cristalização somente das fases esperadas: HT, HAp e NTC. A introdução de NTC na matriz do material melhorou a cristalização das fases HT e HAp, apresentando uma melhor definição e intensidade dos picos registrados e mantendo praticamente inalterados os volumes e parâmetros de célula unitária das fases. Todas as amostras apresentaram cristais variando entre 1 e $5 \mu \mathrm{m}$. Dados de EDS sugeriram que a fase HT não foi afetada pela introdução de $1 \%$ de NTC na matriz do material, uma vez que as amostras HTHAp e HTHAp1NTC exibiram razões catiônicas estequiométricas $(\mathrm{Mg} / \mathrm{Al} \approx 2,0$ e $\mathrm{x} \approx 0,32)$. As amostras HTHAp5NTC e HTHAp15NTC apresentaram variações importantes nas mesmas razões catiônicas, provavelmente devido às interações iônicas entre as fases precursoras. Os modos vibracionais FT-IR e Raman das amostras sintetizadas foram identificados e estão em concordância com as bandas de absorção referentes aos grupos inorgânicos $\left(\mathrm{CO}_{3}{ }^{2-}\right.$, $\left.\mathrm{Al}, \mathrm{Mg}-\mathrm{O}, \mathrm{PO}_{4}^{3-}, \mathrm{OH}^{-}\right)$e moléculas de $\mathrm{CO}_{2}$ e $\mathrm{H}_{2} \mathrm{O}$ presentes nas fases HT e HAp. Dados de FT-IR não registraram os sinais referentes aos NTC, enquanto que as bandas D e G foram observadas via Raman. O aumento do teor de NTC na matriz do material diminuiu a intensidade das bandas em ambas as técnicas espectroscópicas, entretanto não foram observados deslocamentos significativos nas faixas de frequências das mesmas. Dados de TG/DTA de todas as amostras apresentaram três etapas endotérmicas principais, correspondentes aos eventos térmicos de: 1) desidratação das fases HT e HAp; 2) início dos eventos concomitantes de desidroxilação e descarbonatação da fase HT; e 3) término das perdas dos grupos $\mathrm{OH}^{-}$e $\mathrm{CO}_{3}^{2-}$, juntamente com a decomposição dos NTC. A amostra HTHAp15NTC exibiu a maior perda de massa (49\%). A introdução de NTC na matriz dos materiais sintetizados contribuiu significativamente para o aumento dos parâmetros $\mathrm{S}_{\mathrm{BET}}, \mathrm{V}_{\mathrm{P}}$ e $\mathrm{D}_{\mathrm{P}}$ nas amostras dopadas, consequentemente melhorou o rendimento da reação de transesterificação. A amostra testada (HTHAp1NTC) apresentou conversões em ésteres metílicos de $35,2 \%(\mathrm{p} / \mathrm{p})$ a $180{ }^{\circ} \mathrm{C}$ e de $40,5 \%(\mathrm{p} / \mathrm{p})$ a 240 ${ }^{\circ} \mathrm{C}$, comprovando a estabilidade térmica do material dopado e aptidão para ser utilizado como catalisador.

\section{REFERÊNCIAS}

[1] H. Ji, W. Wu, F. Li, X. Yu, J. Fu, L. Jia, J. Hazard. Mater. 334 (2017) 212.

[2] H. Hase, T. Nishiuchi, T. Sato, T. Otake, T. Yaita, T. Kobayashi, T. Yoneda, J. Hazard. Mater. 329 (2017) 49.

[3] A. Salama, J. Colloid Interface Sci. 487 (2017) 348.

[4] M.E. Borges, L. Díaz, Renew. Sustain. Energy Rev. 16 (2012) 2839.

[5] I. Reyero, I. Velasco, O. Sanz, M. Montes, G. Arzamendi, L.M. Gandía, Catal. Today 216 (2013) 211.

[6] Z. Helwani, M.R. Othman, N. Aziz, W.J.N. Fernando, J. Kim, Fuel Process. Technol. 90 (2009) 1502.

[7] L.J. Konwar, J. Boro, D. Deka, Renew. Sustain. Energy Rev. 29 (2014) 546.

[8] F.E. Kiss, M. Jovanovic, G.C. Boskovic, Fuel Process. Technol. 91 (2010) 1316.

[9] A.F. Lee, J.A. Bennett, J.C. Manayil, K. Wilson, Chem. Soc. Rev. 43 (2014) 7887.

[10] Y. Ma, Q. Wang, L. Zheng, Z. Gao, Q. Wang, Y. Ma, Energy 107 (2016) 523.

[11] S. Ogo, A. Onda, K. Yanagisawa, Appl. Catal. A Gen. 402 (2011) 188.

[12] C. Ngamcharussrivichai, P. Nunthasanti, S. Tanachai, K. Bunyakiat, Fuel Process. Technol. 91 (2010) 1409.

[13] A. Obadiah, G.A. Swaroopa, S.V. Kumar, K.R. Jeganathan, A. Ramasubbu, Bioresour. Technol. 116 (2012) 512.

[14] J.A. Rivera, G. Fetter, L. Baños, J. Guzmán, P. Bosch, J. Porous Mater. 16 (2009) 401. 
[15] H. Brasil, P. Pereira, J. Corrêa, L. Nascimento, V. Rumjanek, V. Almeida, N. Coral, E. Rodrigues, Catal. Lett. 147 (2017) 391.

[16] T. Sani, M. Adem, G. Fetter, P. Bosch, I. Diaz, Water, Air, Soil Pollut. 227 (2016) 90.

[17] D. Eder, Chem. Rev. 110 (2010) 1348.

[18] M. Endo, M.S. Strano, P.M. Ajayan, in Carbon Nanotub. Adv. Top. Synth. Struct. Prop. Appl., A. Jorio, G. Dresselhaus, M.S. Dresselhaus (Eds.), Springer, Berlin (2008) 13.

[19] G.-D. Zhan, J.D. Kuntz, J. Wan, A.K. Mukherjee, Nat. Mater. 2 (2003) 38.

[20] Y. Cao, G. Li, X. Li, Chem. Eng. J. 292 (2016) 207.

[21] L. Wang, A. Cao, G. Liu, L. Zhang, Y. Liu, Appl. Surf. Sci. 360 (2016) 77.

[22] P. Serp, M. Corrias, P. Kalck, Appl. Catal. A Gen. 253 (2003) 337.

[23] P.M. Pereira, "Síntese e caracterização do material hidrotalcita-hidroxiapatita e sua aplicação na reação de transesterificação do óleo de soja”, Tese Dr., Un. Fed. Pará (2016).

[24] G. Gelbard, O. Brès, R.M. Vargas, F. Vielfaure, U.F. Schuchardt, J. Am. Oil Chem. Soc. 72 (1995) 1239.

[25] S.Y. Reda, B. Costa, R. Sossela, Rev. Anal. 34 (2008) 86.

[26] F.M. Labajos, V. Rives, M.A. Ulibarri, J. Mater. Sci. 27 (1992) 1546.

[27] J.T. Kloprogge, D. Wharton, L. Hickey, R.L. Frost, Am. Mineral. 87 (2002) 623.
[28] S.J. Palmer, T. Nguyen, R.L. Frost, J. Raman Spectrosc. 38 (2007) 1602.

[29] S.J. Palmer, R.L. Frost, T. Nguyen, Coord. Chem. Rev. 253 (2009) 250.

[30] G.T. Zhou, Q.Z. Yao, J. Ni, G. Jin, Am. Mineral. 94 (2009) 293.

[31] U.J. Kim, X.M. Liu, C.A. Furtado, G. Chen, R. Saito, J. Jiang, M.S. Dresselhaus, P.C. Eklund, Phys. Rev. Lett. 95 (2005) 157402.

[32] S.K. Sharma, P.K. Kushwaha, V.K. Srivastava, S.D. Bhatt, R.V. Jasra, Ind. Eng. Chem. Res. 46 (2007) 4856.

[33] A. Ślósarczyk, Z. Paszkiewicz, C. Paluszkiewicz, J. Mol. Struct. 744-747 (2005) 657.

[34] S. Koutsopoulos, J. Biomed. Mater. Res. 62 (2002) 600. [35] Z. Marković, D. Kepić, I.H. Antunović, M. Nikolić, M. Dramićanin, M.M. Cincović, B.T. Marković, J. Raman Spectrosc. 43 (2012) 1413.

[36] E.D. Dikio, N.D. Shooto, Chem. Sci. Trans. 2 (2013) 1160 .

[37] S. Lowell, J.E. Shields, M.A. Thomas, M. Thommes, Characterization of porous solids and powders: surface area, pore size and density, Springer, Netherlands (2004).

[38] T.S. Stanimirova, N. Piperov, N. Petrova, G. Kirov, Clay Miner. 39 (2004) 177.

[39] H. Eslami, M. Solati-Hashjin, M. Tahriri, F. Bakhshi, Mater. Sci. Pol. 28 (2010) 5.

[40] A. Misra, P.K. Tyagi, M.K. Singh, D.S. Misra, Diam. Relat. Mater. 15 (2006) 385.

(Rec. 13/03/2017, Rev. 12/06/2017, Ac. 15/08/2017) 